\section{P1-404 THYROID CANCER INCIDENCE IN BRAZIL: AN APPROACH USING POLYNOMIAL MODELS}

doi:10.1136/jech.2011.142976f.94

${ }^{1} \mathrm{~A}$ Brito, ${ }^{*} \mathrm{C}$ Coeli, ${ }^{2} \mathrm{~F}$ Barbosa, ${ }^{2} \mathrm{R}$ Caetano, ${ }^{4} \mathrm{M}$ Santos, ${ }^{3} \mathrm{M}$ Vaisman. ${ }^{1}$ Fluminense Federal University, Rio de Janeiro, Brazil; ${ }^{2}$ State University of Rio Janeiro, Rio de Janeiro, Brazil; ${ }^{3}$ Federal University of Rio de Janeiro, Rio de Janeiro, Brazil; ${ }^{4}$ Brazilian National Cancer Institute, Rio de Janeiro, Brazil

Introduction Thyroid cancer represents almost $1 \%$ of malignant neoplasms, corresponding to $0.5 \%$ of total deaths caused by cancer. Despite a low and progressive reduction in mortality rate, thyroid cancer is a public health issue because of its increasing incidence, as shown in various studies worldwide.

Methods This ecological study to estimate thyroid cancer incidence according to age and sex in Brazil in 2006. We employed polynomial models, which take into account age and birth cohort effects in estimate process.

Results We estimated 6066 new cases of thyroid cancer, of which 1065 among men and 5001 among women. The incidence rates were, respectively, 1.16 and 5.27 per 100000 inhabitants. There was an increase in rates according to age for both sexes, especially from 30 years old onwards.

Conclusion The rates' magnitude and profile according to age and sex suggest the method employed yields plausible estimates.

\section{P1-405 JOB STRESS, WORKPLACE SOCIAL SUPPORT AND OCCUPATIONAL ACCIDENTS IN THE PRO-SAUDE STUDY, BRAZIL}

doi:10.1136/jech.2011.142976f.95

\begin{abstract}
${ }^{1}$ A Brito, ${ }^{*}{ }^{1,2}$ A C P de Leon, ${ }^{1,2} \mathrm{G}$ Werneck, ${ }^{1,3} \mathrm{D}$ Chor, ${ }^{1,2} \mathrm{E}$ Faerstein, ${ }^{1,2} \mathrm{C}$ Lopes. ${ }^{1}$ Fluminense Federal University, Rio de Janeiro, Brazil; ${ }^{2}$ State University of Rio de Janeiro, Rio de Janeiro, Brazil; ${ }^{3}$ Oswaldo Cruz Foundation, Rio de Janeiro, Brazil
\end{abstract}

Introduction The objective of this study was to investigate the association between job stress and the occurrence of occupational accidents using multilevel modelling in order to take into account clustering of participants at their workplaces.

Methods Analyses were based on 3572 university employees $\leq 70$ years of age during the second phase of data collection of a longitudinal study (Estudo Pró-Saúde). The period of recall for accidents was the 12 month before answering the self-reported questionnaire. Psychosocial stress at work was approached considering the interplay between high psychological demand and low control over the labour process. These dimensions were taken by means of the summarised version of the Karasek scale, which also contains information about the social support at work. Isolated dimensions of job stress (demand and control), the ratio between scores of psychological demand and control over the work process (ratio D/C) and social support at work were measured at individual level and at the level of the workplace.

Results The total prevalence of accidents in the 12 month period was $26 \%$. Results indicate that high psychological demands measured at the individual level was an important factor associated to the occurrence of occupational accidents, particularly among those with scores above the population average, independently of the level of control that the subject might have over the work process. Social support at work was inversely associated to the prevalence of occupational accidents. At the workplace level, this association was characterised by a curvilinear relationship more evident among female worker.

\section{P1-406 CHARACTERISTICS OF INDIVIDUALS “LEFT BEHIND” IN DEPRIVED AREAS IN SCOTLAND}

doi:10.1136/jech.2011.142976f.96

${ }^{1} \mathrm{D}$ Brown, ${ }^{*} \mathrm{D}$ O'Reilly, ${ }^{3} \mathrm{~V}$ Gayle, ${ }^{1} \mathrm{~S}$ Macintyre, ${ }^{1} \mathrm{M}$ Benzeval, ${ }^{1} \mathrm{~A} \mathrm{H}$ Leyland. ${ }^{1} \mathrm{M} R \mathrm{C} /$ CSO SPHSU, Glasgow, UK; ${ }^{2}$ Queen's University, Belfast, UK; ${ }^{3}$ University of Stirling, Stirling, UK

Introduction Previous work in Scotland has shown that within deprived areas, declining populations have higher illness and mortality rates than stable or increasing populations. One possible explanation is that as healthy individuals move out, they are replaced by individuals in poorer health or not replaced at all, producing areas with higher concentrations of poor health. Little is currently known about the characteristics of migrants to and from these deprived areas or the populations remaining.

Methods Using data from the Scottish Longitudinal Study (SLS), which links individual level Scottish census data to vital events, we examine the socio-demographic characteristics and health status of individuals aged 16-74 who moved in and out of deprived areas with declining populations in the year prior to the 2001 census. We also examine the characteristics of those left behind.

Results 1771 SLS members remained living in a deprived, declining area while 242 individuals moved in and 466 moved out. Compared to the residentially stable, those moving were more likely to be from a non-White ethnic group $(p<0.001)$, unemployed but seeking work $(p=0.001)$ and single $(p<0.001)$ while those who moved out were more likely to be from a higher social class $(p<0.001)$. Rates of poor general health, long term illness and some causes of death were highest for those who remained in these areas.

Conclusion People who remain in deprived and declining areas have a different socio-demographic and health profile from those who move into and out of these areas; non-migrants were in worst overall health.

\section{P1-407 THE EMERGING EPIDEMIC OF OBESITY IN PUNJAB (INDIA): A POPULATION BASED STUDY}

doi:10.1136/jech.2011.142976f.97

R Soni,* G Wander, N Gupta. Dayanand Medical College and Hospital, Ludhiana, Punjab, India

Introduction Obesity is a complex, multifactorial chronic disease involving genetic, physiological, metabolic, behavioural, psychological and environmental components. The global epidemic of overweight and obesity - "globesity" - is rapidly becoming a major public health problem. It has reached epidemic proportion in India in the $21^{\text {st }}$ century.

Methods The present population based study has been conducted in rural and urban areas of Ludhiana district, Punjab State (India). The study included 1615 Punjabi adults comprising 875 from rural and 740 from urban area. Demographic variables (age, sex, SES, lifestyle) and anthropometric parameters-height, weight, waist circumference and hip circumference were recorded. Biochemical analysis viz. cholesterol, $\mathrm{Na}$ and $\mathrm{K}$ were also done.

Results Out of 1615 subjects, 44\% were males and $56 \%$ were females. Half of the subjects were in the age group of 20-40 years. Overall prevalence of overweight and obesity has been found be $18.8 \%$ and $46.6 \%$, respectively. Prevalence of obesity is slightly higher in rural subjects than the urban. The percentage of obese subjects is $54 \%, 55.35 \%$ and $56.44 \%$ in the age groups of $\leq 30$ years, $31-40$ years and $41-50$ years, respectively. Prevalence of obesity has been computed in relation to sex, SES and lifestyle. Serum 
cholesterol level was significantly $(\mathrm{p}=0.007)$ higher among obese (192.24 \pm 41.39$)$ than non obese $(179.27 \pm 39.57)$ subjects. No significant difference of $\mathrm{Na}$ and $\mathrm{K}$ was observed between obese and non obese subjects.

Conclusion The prevalence of obesity is alarmingly high in adult population of Punjab.

\section{P1-408 PREGNANCY PLANNING AND THE ROLE OF SOCIAL INEQUALITIES IN HEALTH AND DEVELOPMENTAL OUTCOMES AT 5 YEARS}

doi:10.1136/jech.2011.142976f.98

${ }^{1} \mathrm{C}$ Carson, ${ }^{*}{ }^{2} \mathrm{~A}$ Sacker, ${ }^{1} \mathrm{M}$ Redshaw, ${ }^{2} \mathrm{Y}$ Kelly, ${ }^{1} \mathrm{~J}$ Kurinczuk, ${ }^{1} \mathrm{M}$ Quigley. ${ }^{1}$ National Perinatal Epidemiology Unit, University of Oxford, Oxford, UK; ${ }^{2}$ Institute for Social and Economic Research, University of Essex, Colchester, Essex, UK

Objective To examine the effects of unplanned pregnancy on cognitive, behavioural and health outcomes of children at 5 years.

Design Population-based cohort (Sweep 1 and 3 of the UK Millennium Cohort Study).

Participant 13529 singletons, born 2000-2001.

Exposure measure Mothers reported whether their pregnancy was planned, and their feelings when first pregnant. The population was divided into "unplanned" pregnancies (unplanned and unhappy), "mistimed" pregnancies (unplanned but happy) and "planned".

Outcome measures At 5 years mothers answered questions about their child's health and behaviour (Strength and Difficulties Questionnaire). Children completed cognitive tests (British Abilities Scales II), including a component to measure verbal abilities.

Results $17 \%$ of pregnancies were unplanned, 31\% mistimed, and $52 \%$ planned. On average, children from unplanned pregnancies had cognitive scores indicating a delay of 5 months; were more likely to have behavioural problems (OR 2.9 (95\% CI 2.3 to 3.3)); and more likely to be in poor health than the planned group (OR 2.3 (95\% CI 1.8 to 3.0)). After adjustment the effects were reduced, though remained significant for behavioural and health outcomes: OR 1.3 (95\% CI 1.1 to 1.6$)$ and OR 1.6 (95\% CI 1.2 to 2.1 ), respectively. The effects were smaller in the mistimed group, and after adjustment for confounding by socioeconomic and demographic factors, these effects disappeared.

Conclusions While the adverse outcomes associated with mistimed pregnancies can be explained by confounding of socioeconomic circumstances, there remains a significant effect on the health and behaviour of children born after unplanned pregnancies. Potential confounding by other contextual or family factors will be examined.

\section{P1-409 DIFFERENTIAL LOSS OF PARTICIPANTS DOES NOT NECESSARILY CAUSE SELECTION BIAS}

doi:10.1136/jech.2011.142976f.99

K Carter, ${ }^{*}$ F I Gunasekara, S Mckenzie, T Blakely. Health Inequalities Research Programme, Department of Public Health, University of Otago, Wellington, New Zealand

Background Most research is affected by differential participation, where individuals who do not participate have different character- istics to those who do. This is often assumed to induce selection bias. However, selection bias only occurs if the exposure-outcome association differs for participants compared to non-participants. We empirically demonstrate that selection bias does not necessarily occur when there is varying participation in a study.

Methods We used data from the first three waves of the longitudinal Survey of Family, Income and Employment (SoFIE). We examined baseline associations of labour market activity and education with self-rated health using logistic regression in four participation samples: A) the original sample at year one ( $\mathrm{N}=22265)$; $\mathrm{B})$ drop outs over 3 years $(n=3880)$; C) those remaining in the sample over 3 years $(n=18350)$; and $D)$ those (year 3 ) consenting to further data linkage $(n=14340)$.

Results Loss to follow-up was more likely among lower socioeconomic groups and those with poorer health. However, for labour market activity and education, the odds of reporting fair/poor health were similar across all samples, including after adjustment for sociodemographic variables. Thus, there was little evidence of selection bias.

Conclusions Differential loss to follow-up did not lead to selection bias in the association between socioeconomic measures and selfrated health. When assessing the possibility of selection bias researchers should consider whether differential participation affects the exposure-outcome association, not just participation by exposure or outcome separately.

\section{P1-410 EVALUATION OF INFANT MORTALITY RISK FACTORS IN AN EASTERN AREA OF IRAN; A CASE-CONTROL STUDY- 2009}

doi:10.1136/jech.2011.142976f.100

R Chaman, ${ }^{*}$ P Zolfaghari, M G Taramsari, M B Sohrabi. Shahroud University of Medical sciences, Shahroud, Iran

Introduction Infant Mortality Rate (IMR) is one of the most important indicators of community health situation and especially children health status. This study was conducted for determination of IMR risk factors in Shahroud Township (which is located in the east of Iran).

Methods This study was carried out as a Case- Control study and all infant deaths (117 cases) from 2007 to 2009 in Shahroud Township were selected. For each case two controls were entered the study by matching on time and place of birth (234 controls). After data collection of all related variables, data analysis was performed using relevant statistical methods (Univariate and multivariate conditional logistic regression).

Results Univariate analysis of risk factors showed statistical significance ( $\mathrm{P}$ Value $<0.05$ ) between infant mortality and low birth weight $(<2500 \mathrm{~g})$ with $\mathrm{OR}=15.14$, prematurity ( $<37$ weeks of gestational age) with $\mathrm{OR}=9.85$ and high risk pregnancy with $\mathrm{OR}=3.13$. Multivariate analysis resulted in significant statistical association ( $p$ value $<0.05$ ) between infant mortality and low birth weight $(\mathrm{OR}=8.04)$ and prematurity $(\mathrm{OR}=3.51)$.

Conclusion Based on our findings Low Birth Weight (LBW) and prematurity are the most important risk factors for infant mortality and prevention of these two risk factors will reduce a majority of infant mortalities. Determination of underlying causes of LBW and prematurity needs serious attention of public health investigators. 